\title{
Quantitative and Qualitative Analysis of the Learning Analytics and Knowledge Conference 2018
}

\author{
Xavier Ochoa ${ }^{1}$, Agathe Merceron ${ }^{2}$ \\ ${ }^{1}$ Email: xavier.ochoa@nyu.edu Address: New York University - Steinhardt, 82 Washington Square East, 7th Floor, New York, NY, 10003, USA \\ ORCID ID: 0000-0002-8709-5780 \\ ${ }^{2}$ Email: merceron@beuth-hochschule.de Address: Beuth University of Applied Sciences Berlin, Luxemburger Strasse 10, 13353 Berlin, \\ Germany ORCID ID: 0000-0003-1015-5359
}

\section{Introduction}

LAK-18, the 8th International Conference on Learning Analytics and Knowledge, was hosted by the University of Sydney, Australia, from March 5 to 9, 2018. Traditionally, authors of papers that have received high scores through the review process of the conference are invited to extend their paper and submit it for a special issue of this journal. The chairs of LAK-18 have adopted a double-blind review process that has proven useful in maintaining the technical quality of the conference. Papers submitted to the special issue undergo a rigorous review process that is blind only, not double-blind, as a shorter version of the paper has been already published. The theme for LAK-18 was "Towards User-Centred Analytics". Though several of the papers receiving high scores in the review process explicitly dealt with this issue, it has been decided to devote a complete special issue of the journal to this theme and invite those papers in this special issue to come. Thus, the present special issue LAK-18 covers a broad range of themes, which reflect well the diversity and the vibrancy of the community, but excludes User-Centred Analytics.

In this editorial, we describe the story behind this special issue. This story begins with the contributions submitted to the conference and finishes with a short introduction to the papers in this issue. The two first episodes of the story, contribution type and country, include both tracks, research and practitioner. The reminder episodes refer only to the research track.

\section{Quantitative Analysis of LAK-18}

A conference can be first measured and understood by its numbers. The following section presents key metrics of LAK-18, from the submission of articles, their review, acceptance, and the final program.

\section{Submissions}

The papers accepted into a conference are, by the time and space limitations of the event, just a selection of the work done in the field. To better appreciate the breadth of Learning Analytics research, a study of the unfiltered submissions is presented. A total of 348 works or proposals were submitted to LAK-18. The following subsections decompose those submissions into several categories according to their type, country, topic and methodology used.

\section{Submissions per Contribution Type}

The most immediate division of submission is by their contribution type. Each work was submitted specifically to one type. This year, LAK had seven contribution types. The first type is full papers, scientific articles that include a clearly explained substantial conceptual, technical or empirical contribution to the field. This year 115 full papers were received. The second most popular type is short papers, scientific articles that address on-going work, which may include a briefly described theoretical underpinning, an initial proposal or rationale for a technical solution, and preliminary results. A total of 89 short papers were received. A new type this year is extended abstracts. This type, for some scholarly communities, is a more familiar form of conference submission that can be used as a means for eliciting formative feedback on an idea or initial work. LAK-18 received 23 extended abstracts. A part of LAK, since 2015, is the inclusion of a practitioner track with their own type of submissions. The primary goal of the practitioner track is to share thoughts and findings that stem from learning analytics project implementations in the hope that such conversations will contribute to a shared, discipline understanding of interventions, and the factors affording or constraining their success and take up. This year, 38 practitioner contributions were received. The poster type is a concise report of recent findings or other types of innovative work not ready to be submitted as a full or short paper. A total of 28 posters were received. LAK, since its second edition in 2012, accepts proposals for workshops and tutorials or seminars that are hosted by the conference. Workshops provide an efficient forum for community building, sharing of perspectives, and idea generation for specific and emerging research topics or viewpoints. Tutorials aim to educate stakeholders on a specific 
learning analytics topic or stakeholder perspective. A total of 20 workshops and tutorials were submitted to LAK-18. Finally, LAK host a Doctoral Consortium since 2013. The Doctoral Consortium is a one-day workshop to support emerging scholars in learning analytics by helping them develop productive approaches to studying the intersection of theory, data, and practice in learning analytics, the learning sciences, data sciences, and human-centered computing. This year a total of 35 students submitted their proposal to be accepted at the consortium. Figure 1 shows the growth of submissions for the LAK conference. From the numbers, it is clear that the number of full and short papers, practitioners papers and workshops/tutorials have been stable since 2016. A yearly growth is perceived in the doctoral consortium (new researchers entering the field). This year the presence of extended abstracts compensated a small decline in the number of posters from last year (an anomaly compared also with previous years).

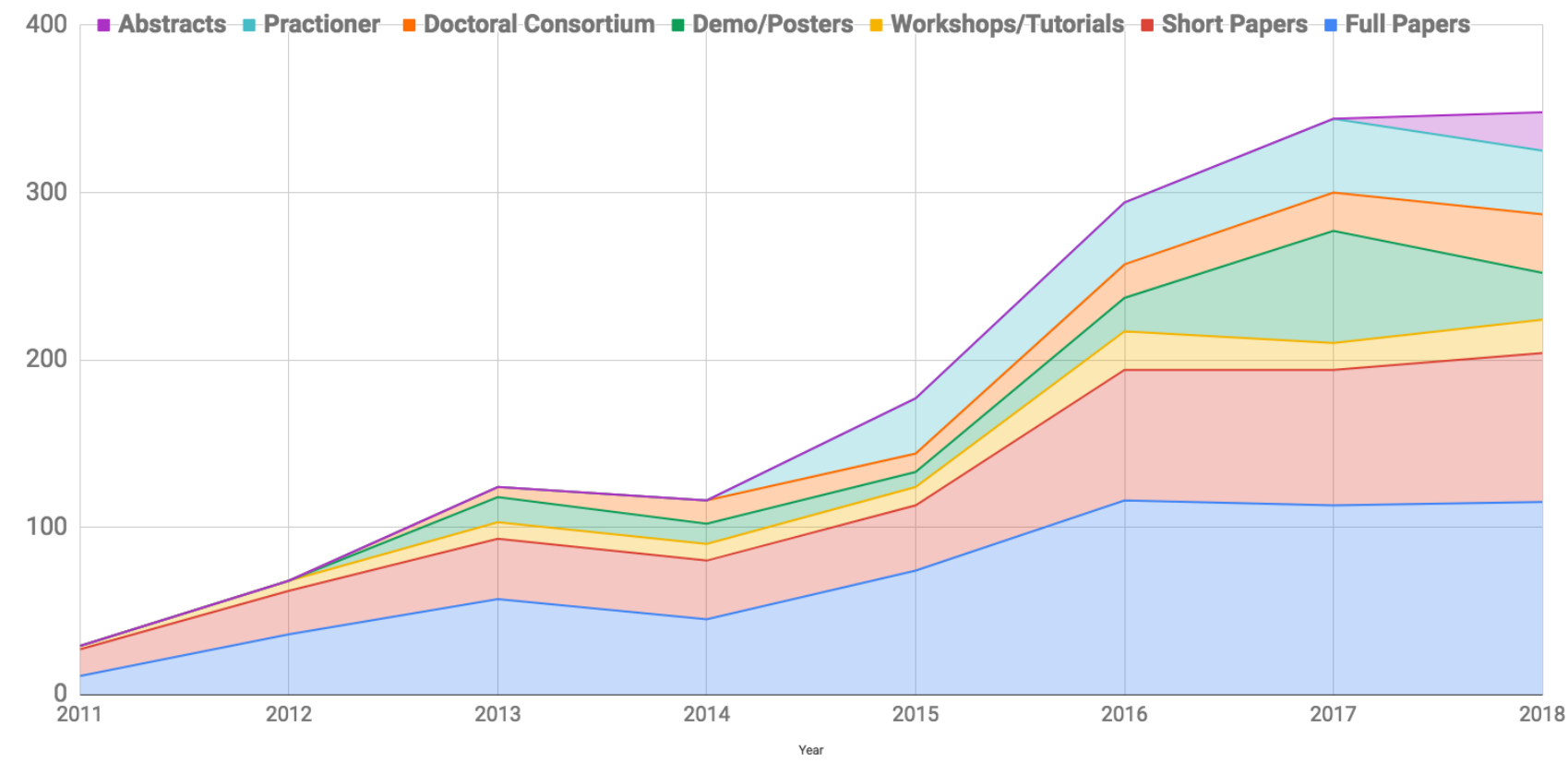

Figure 1. Submissions to LAK by type and year.

\section{Submissions per Country}

LAK is an international conference, not only in intention but also in reality. This year edition received contributions with authors from 29 countries. The top-five contributors were: USA (265 authors), Australia (113), Japan (48), the UK (48) and the Netherlands (36). It is interesting to note that the top-five spots are no longer dominated by Anglo-Saxon countries as in most previous years. The inclusion of Japan and the Netherlands as major players is a welcome change in the field. Also encouraging is the growth in the number of contributors from developing parts of the world such as Latin America, the Middle East, and South-East Asia. An issue, however, that can be seen in Figure 2, is the lack of contributions from African countries (with the exception of South Africa) and Russia. More efforts should be done to include researchers and practitioners from this region into the Learning Analytics discussions.

\section{Submissions per Topic}

When submitted, the authors of full papers, short papers and extended abstracts needed to select what topics their contributions were about. The list of topics were pre-configured by the Program Committee chairs in the submission system (Easychair). The authors could select one or more topics for their submission. Figure 3 shows the number of submissions for each topic listed. The use and evaluation for Learning Analytics tools received most submissions, reflecting the practical nature of Learning Analytics. Also, Learning Analytics is usually used to inform Learning and Instructional Design. Infrastructure is still a very discussed topic, reflecting the needs to have a solid computational system to capture and process educational data. Adaptive Learning has found a home in Learning Analytics (and vice versa), where data processing is commonly used to generate learner models and implement recommenders systems. Finally, a topic that has grown considerably in the last years is the use of Learning Analytics to provide feedback to students and instructors. 


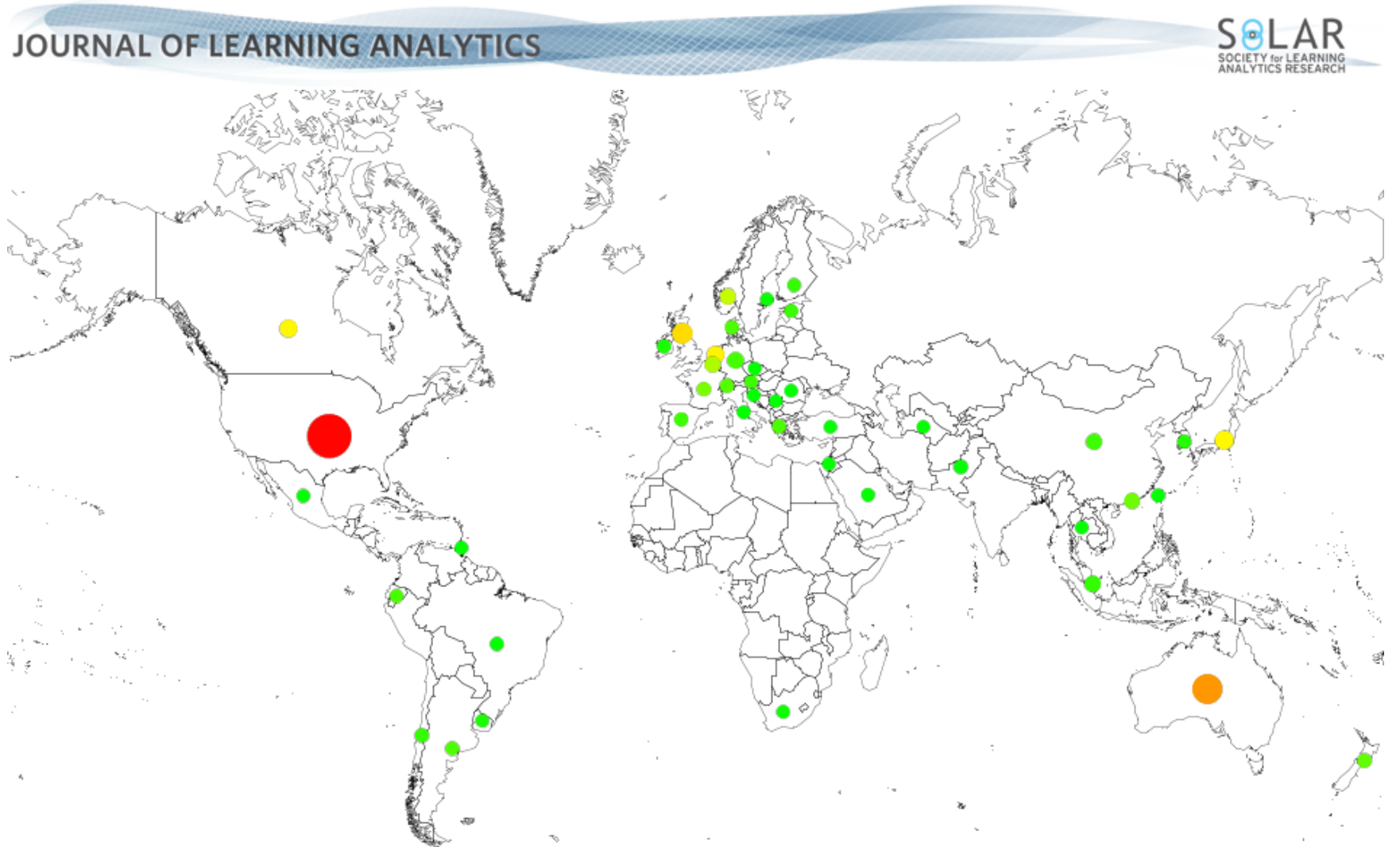

Figure 2. Submissions per country. Node's size and color represent the contribution of that country to the submitted papers.

It is interesting to note that there still a considerable number of papers that discuss the history and future of the Learning Analytics field. This suggests that authors are preoccupied with the field itself and defining its boundaries, opportunities, and development.

\section{Submissions per Method}

The authors of full and short papers and extended abstracts also had to select one or more methods used in their articles. Figure 4 presents the number of submissions that selected each method. Data Mining and Machine Learning are the most used methods submitted to LAK, reflecting our cross-pollination with the Educational Data Mining field. However, statistical analysis and modeling, preferred tools of quantitative Educational Researchers occupy the third and fourth positions. Also encouraging is the presence of qualitative analysis and mixed methods, used in more traditional Educational Research. This plethora of methods is a testament to the multivocality (Suthers \& Verbert, 2013) of Learning Analytics.

\section{Submissions by Topic and Method}

To obtain a better view of the methods used by each topic, a cross tabulation was created and it is presented in Figure 5. There are methods that are heavily used across all topics such as Case Studies, Data Mining, Machine Learning, Inferential Statistics and Statistical Modelling. An interesting cross-topic methodology is Qualitative Analysis, due to the more quantitative nature of the field. Other methodologies are specifically linked to certain topics, for example, Natural Language Processing is mainly used in Understanding Discourse and Multimodal Analytics in Cognition and Memory. Also, there as topics, such as Feedback Systems, that make use of most of the methods.

\section{Review Phase on the Research Track}

The Program Committee of the Research Track of LAK-18 had 85 members, that had the role of senior reviewers. The conference also had 161 reviewers. The country of origin of this reviewers can be seen in Figure 6. It is interesting to note that the geographical distribution of reviewers is similar to the geographic distribution of submission, shown in Figure 1.

This year, each reviewer and senior reviewer were assigned between one and eight research papers, with an average of 2.57 per reviewer. We had an excellent response rate, with $80 \%$ of reviews delivered by the deadline. The $90 \%$ of the reviews submitted was reached in the expanse of an additional week. The submission time of each review response can be seen in Figure 7.

A simple, although very rough, estimation of the quality of the reviews can be obtained by counting the number of words in the review. The average length of the reviews was 326 words, healthy over the required 250 words. The distribution of 


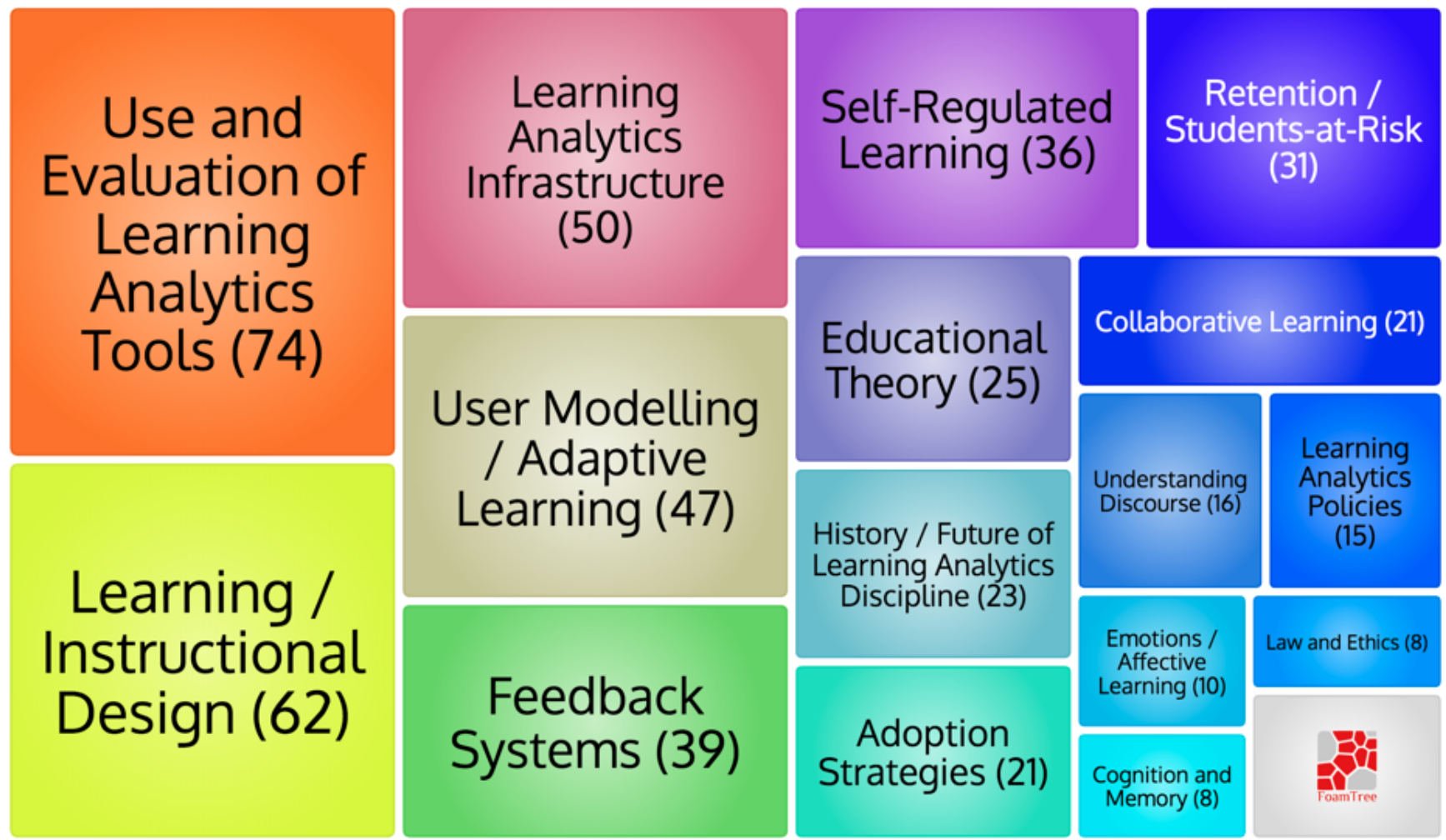

Figure 3. Submissions per topic. Box's size represents the number of submissions in that topic. A submission can be classified in one or more topics.

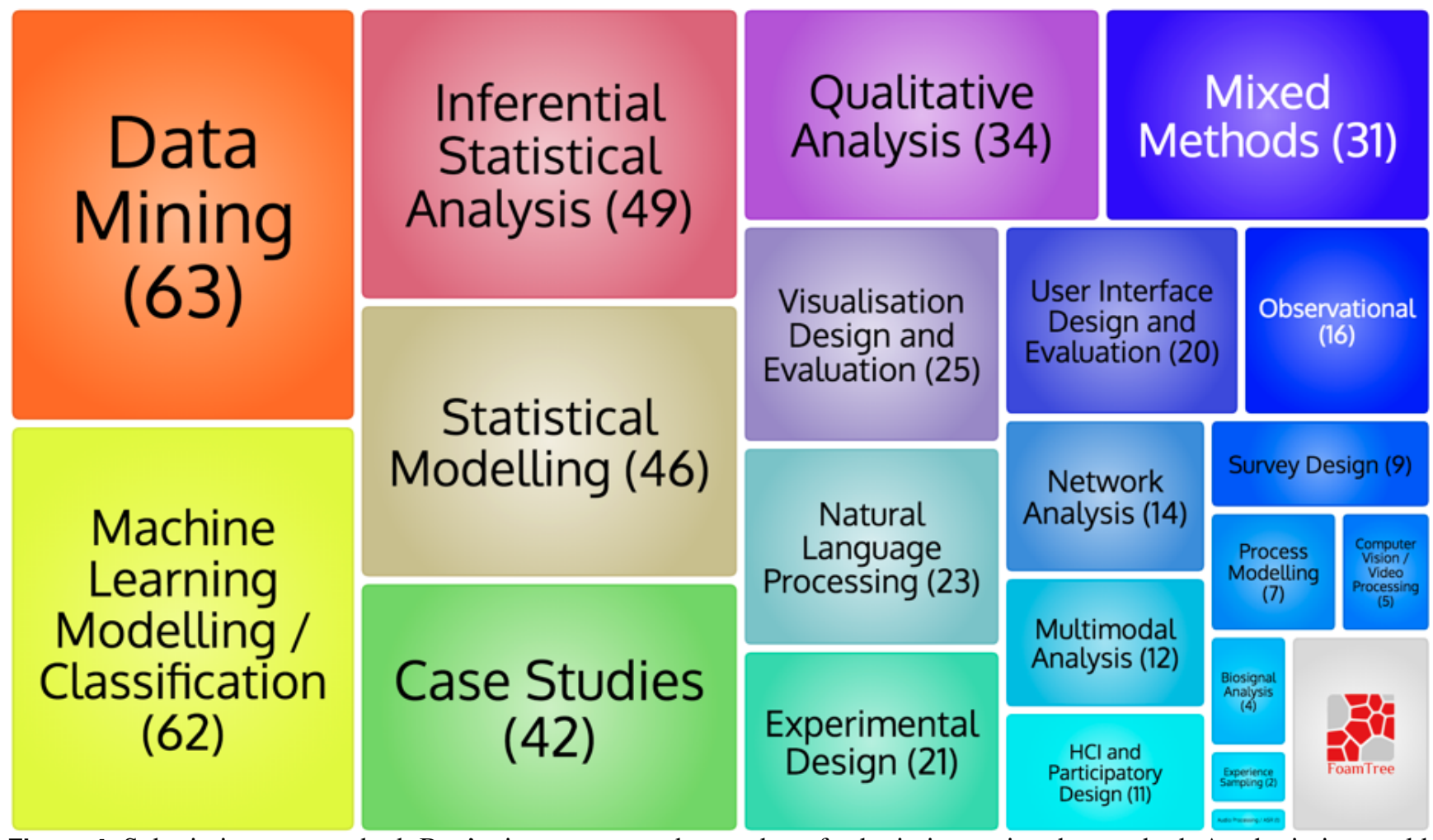

Figure 4. Submissions per method. Box's size represents the number of submissions using that method. A submission could use one or several methods. 


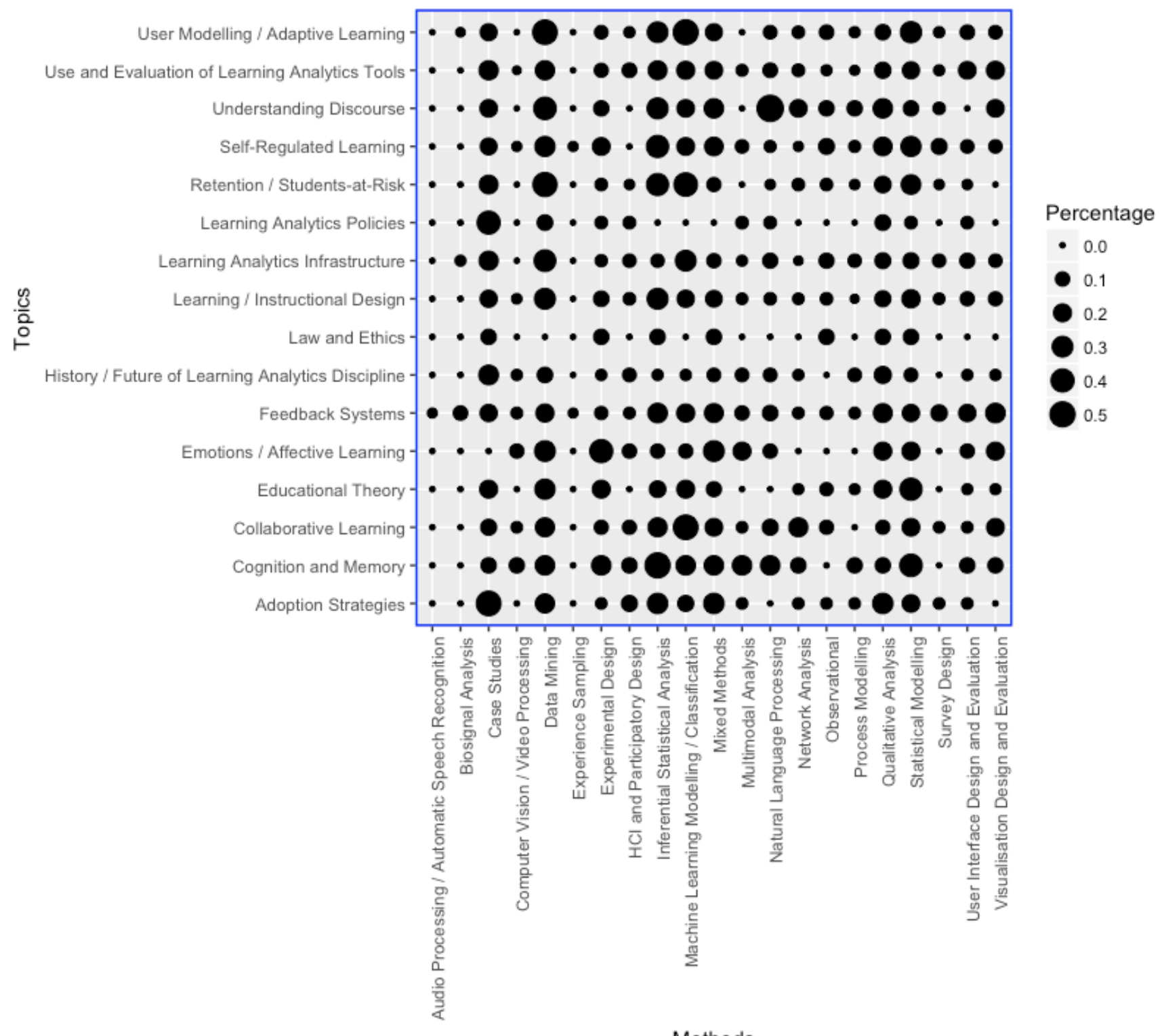

Methods

Figure 5. Submissions per topic and method. Circle's size represents the number of submissions classified in a given topic that use a given method. 


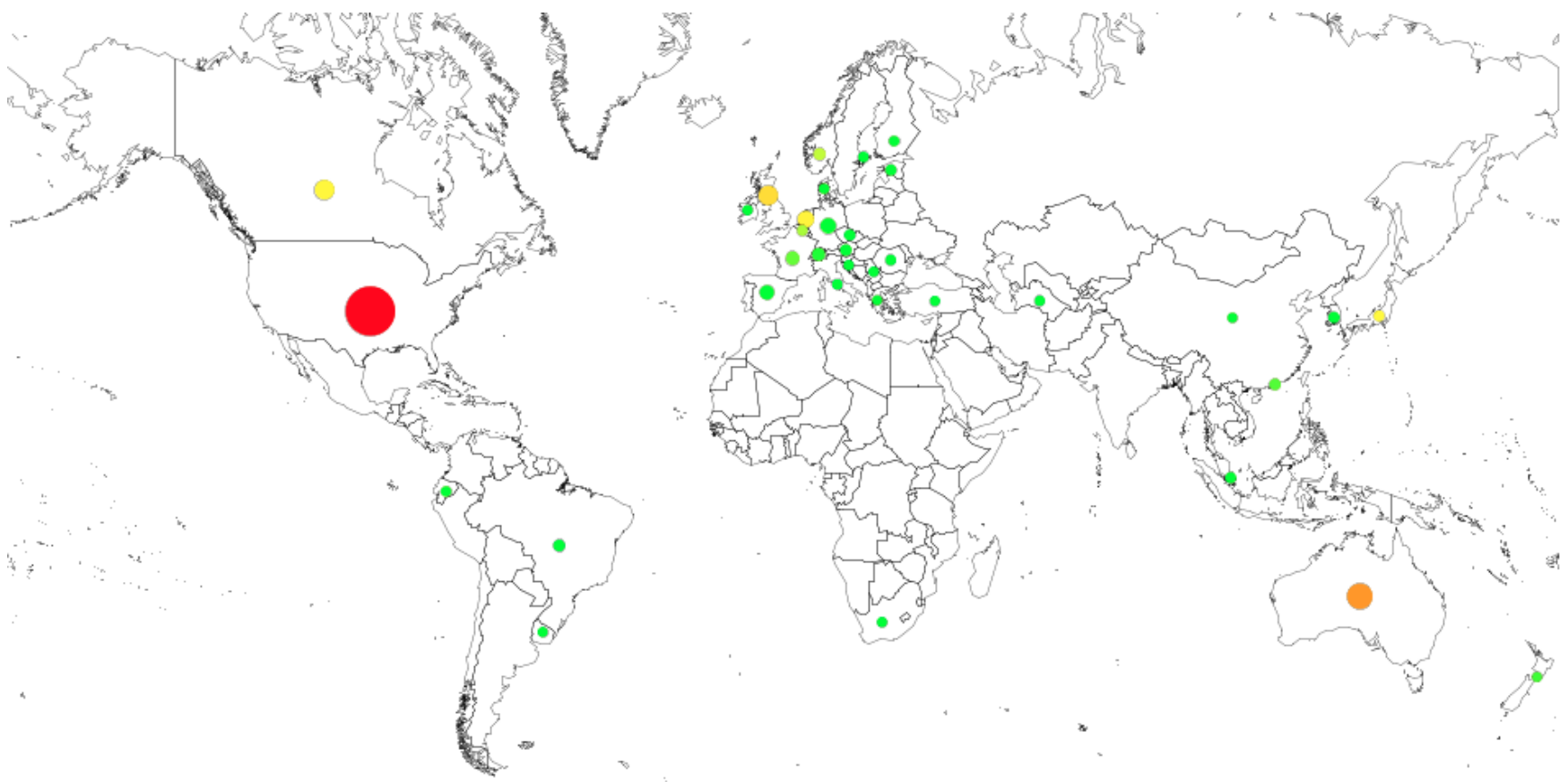

Figure 6. Reviewers per country. Circle's size and color represent the number of reviewers.

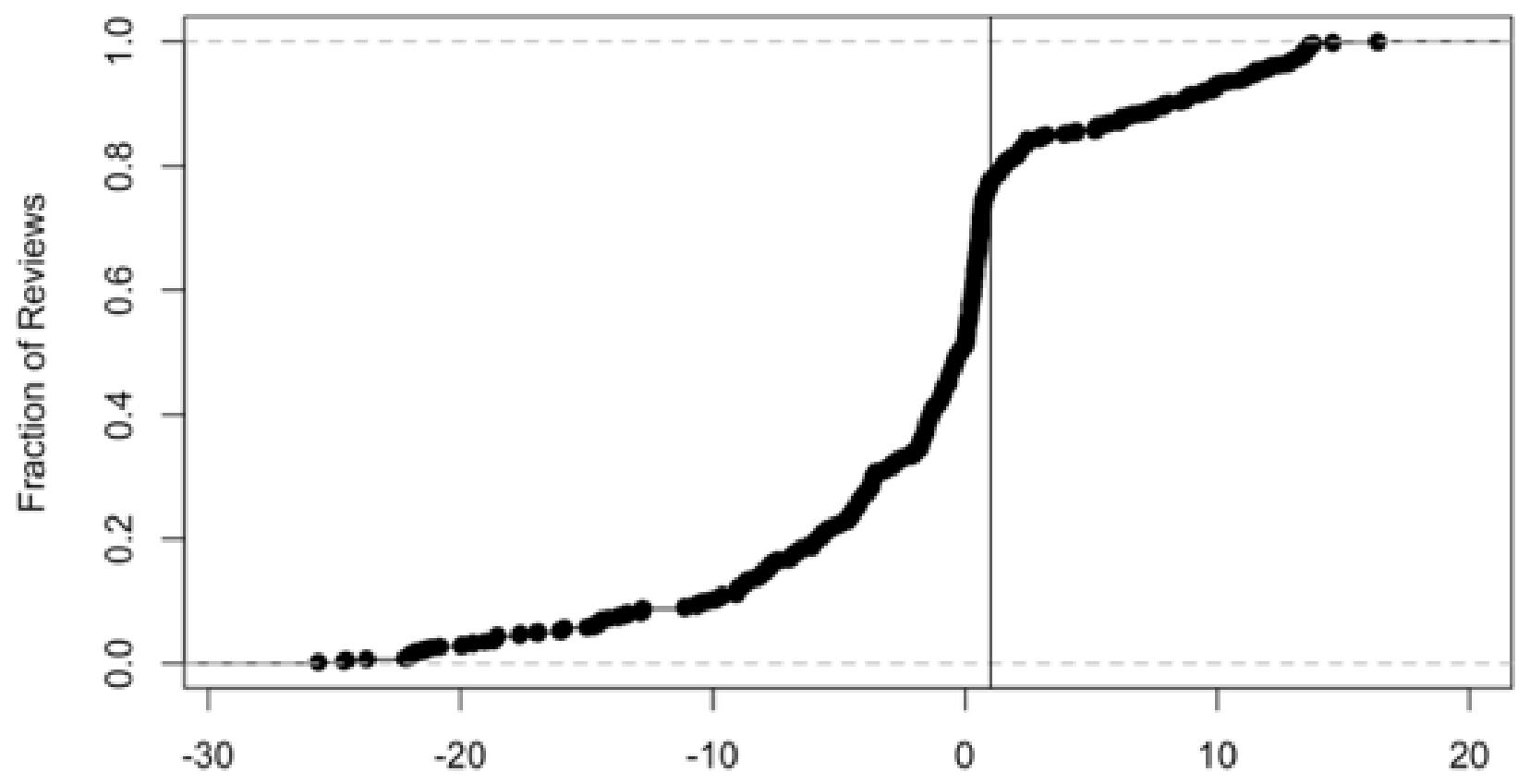

Days before / after Deadline

Figure 7. Time of review submission. Points to the left of the vertical line are on-time submissions. 


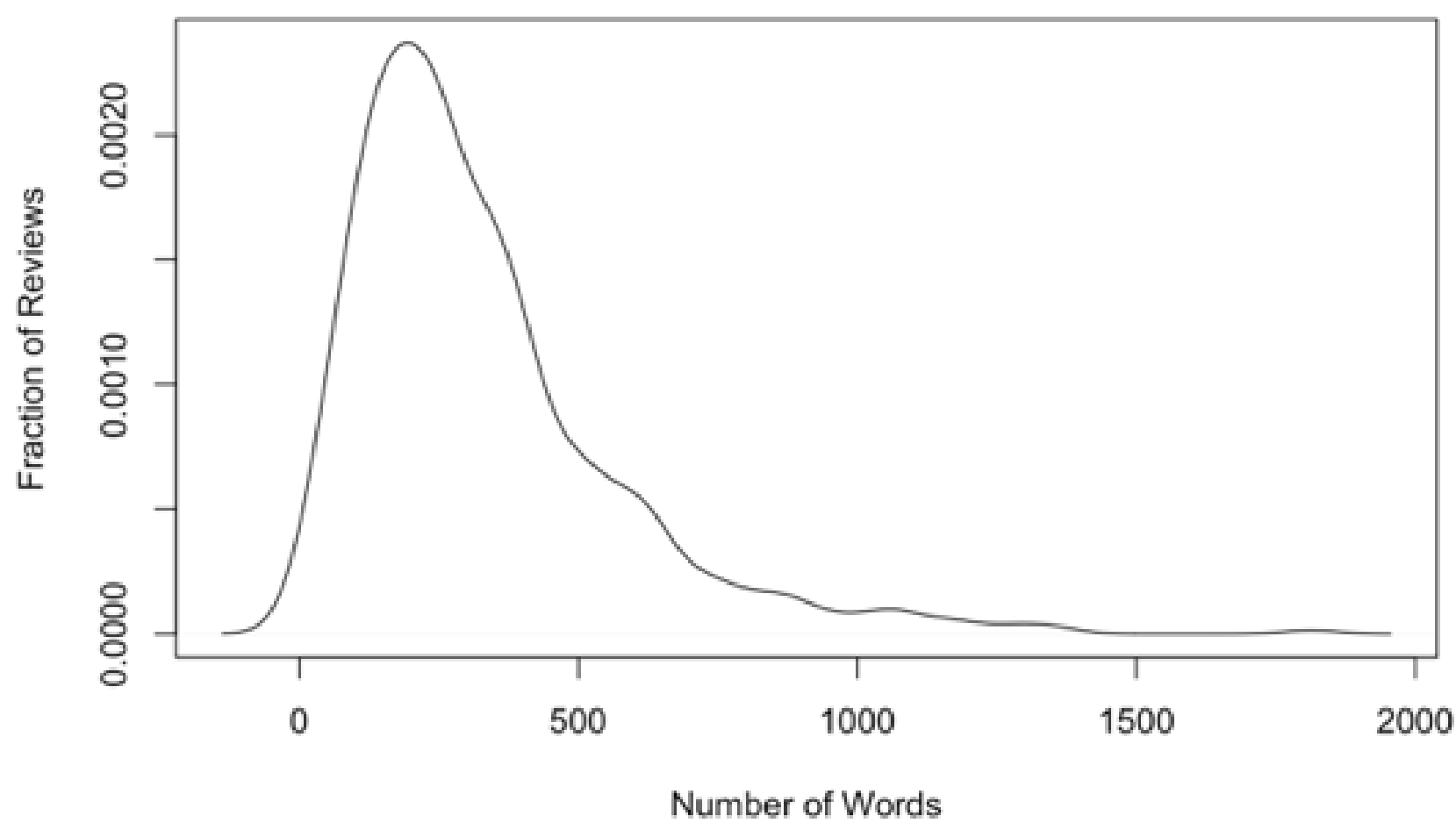

Figure 8. Average number of words per review. Each point represents a reviewer.

these values can be seen in Figure 8. A long tail distribution means that a small group of reviewers provided very detailed explanations to the authors about their review.

An interdisciplinary field, such as Learning Analytics, always presents a challenge to the Program Committee when scores are provided by a diverse group of reviewers. What could be an excellent paper from a computational point of view could be very weak from a more educational perspective and vice versa. This variance in the score could be easily captured by measuring the deviation score of each reviewer from the average score finally given to the paper. In the case of LAK, where papers had 3 reviewers, and each one can score the paper from +3 (Strong Accept) to -3 (Strong Reject), the deviation could take values from +4 (two -3 scores and the reviewer providing a +3 ) to -4 (two +3 scores and the reviewer providing a -3 ). If all the reviewers were in agreement, the deviation score for all of them is 0 . If the deviation for each review is obtained, the distribution shown in Figure 9 appears. Most reviews have a deviation in the range from -1 to +1 . However, there is a sizable portion ( $42 \%)$ than is outside this interval, signaling more diverging opinions in the reviewers.

To help the Program Committee chairs to make a final decision in the face of this diversity of opinions, this year LAK assigned Program Committee members with the task of meta-reviewing the submissions to the Research Track and provide a recommendation of acceptance or rejection to the chairs. This process was very successful and the recommendation of the meta reviewers agreed $93.97 \%$ of the time with the final decision of the chairs. Moreover, this metareview phase help the conference to have a fairer review process that did not rely on reviewers' scores to reach an acceptance decision. Proof of this can be seen in Figure 10, where full papers in the upper middle range of the scores are accepted or rejected independently of this score.

\section{Accepted Papers and Authorship in the Research Track}

From the 227 submissions received to the Research Track, 62 were accepted to be presented at LAK-18: 36 full papers, 22 short papers and 4 extended abstracts. A 30\% acceptance rate for full papers, is very similar to previous editions of the conference. The countries with more contribution to the accepted papers were USA: 22.5, Australia: 12.5, Canada: 4.4, Belgium: 3.1, and the Netherlands: 3.1. However, this picture should be complemented with the notion that $25 \%$ of the papers in the research track are the result of international collaboration as it can be seen in Figure ??.

The authors of the accepted articles form a co-authorship network with special characteristics. Figure 12 presents this co-authorship network. The size of the nodes represent the number of contributions made to any LAK conference. For the smallest nodes this is their first contributions at LAK, while larger nodes are regular and prolific contributors. The color of 


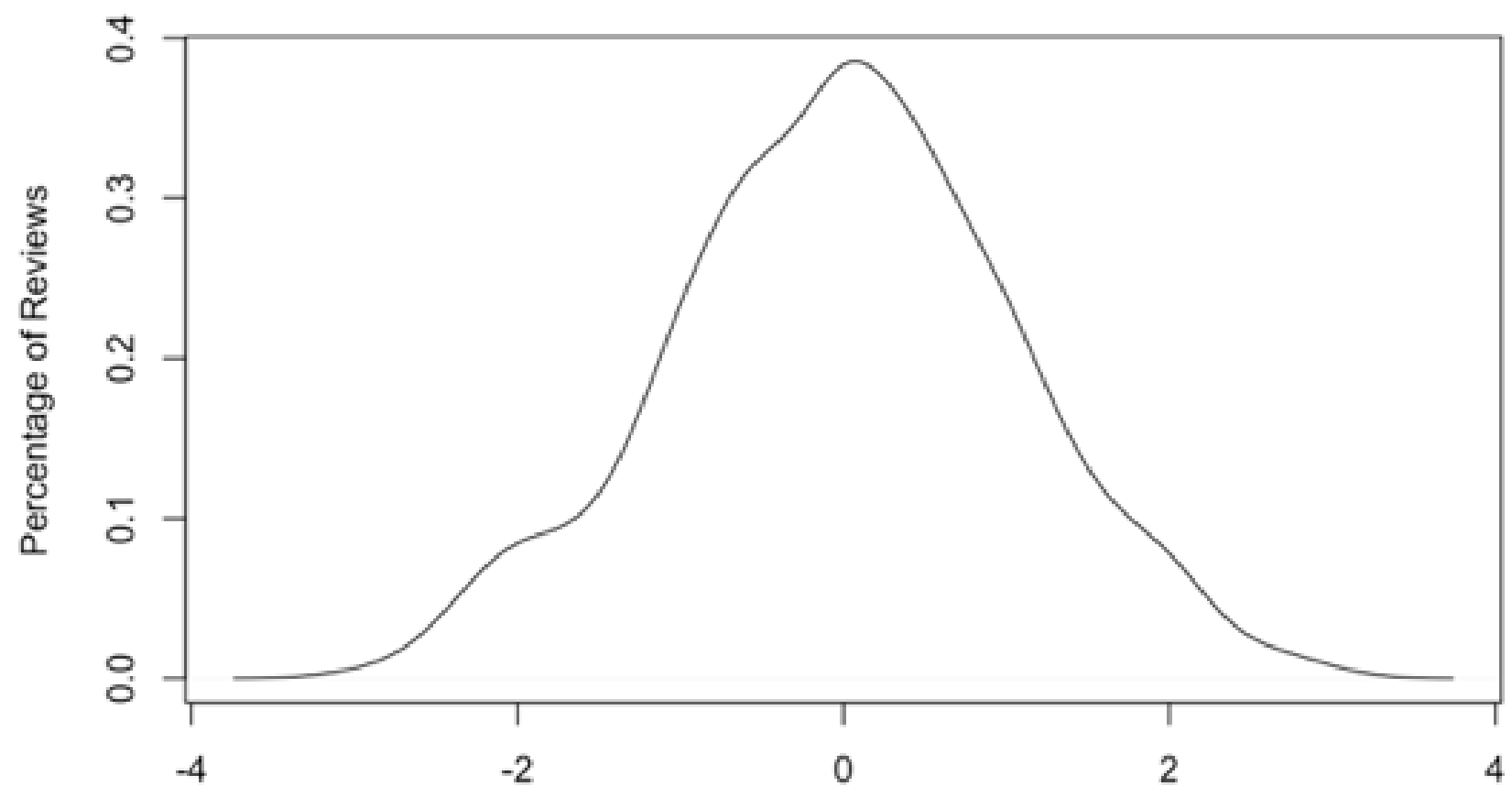

Deviation points

Figure 9. Distribution of average deviation of each reviewer from the final score of the papers.

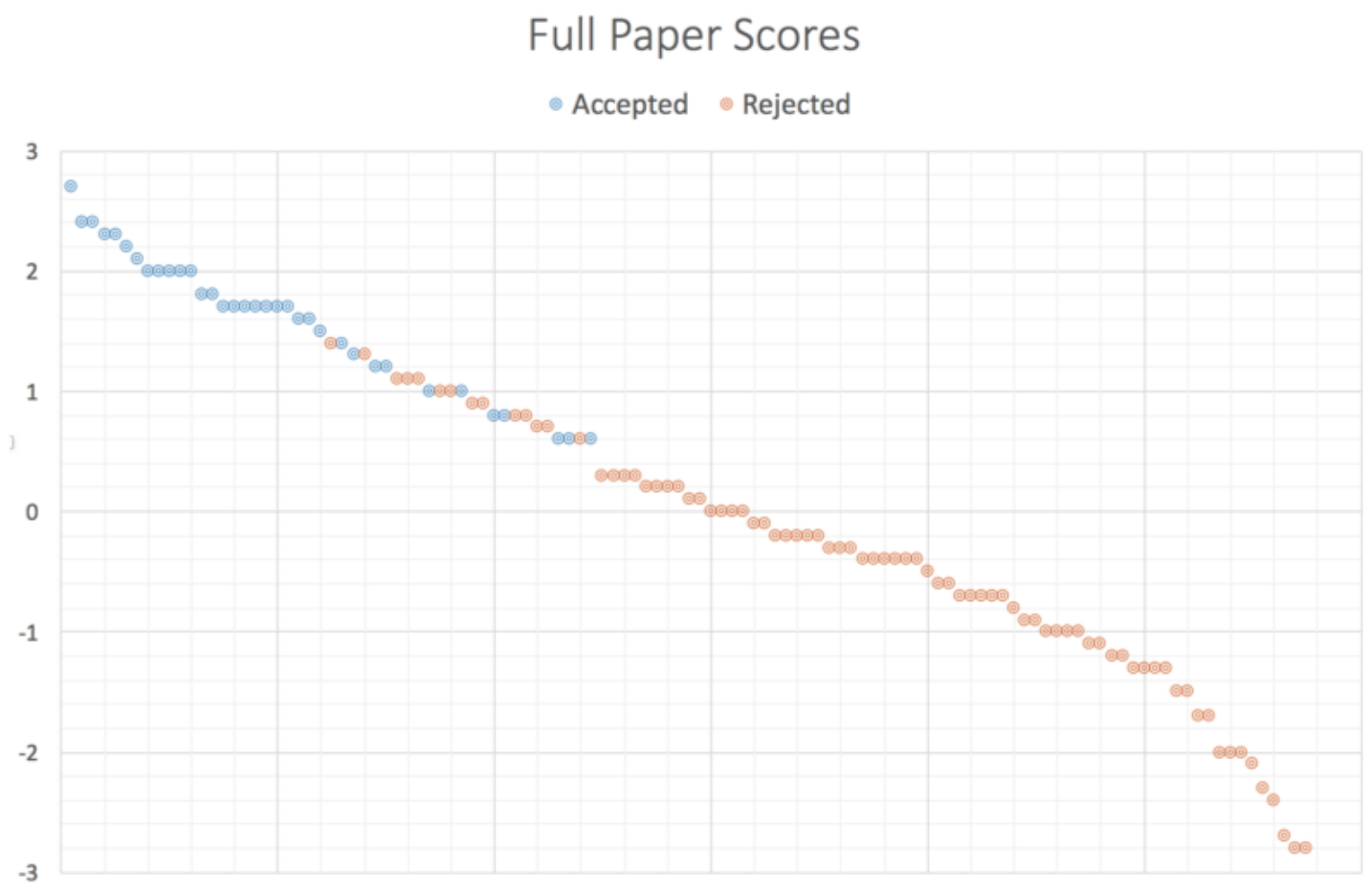

Figure 10. Accepted paper by score. Blue dots represents accepted papers, red dots, rejected papers. 


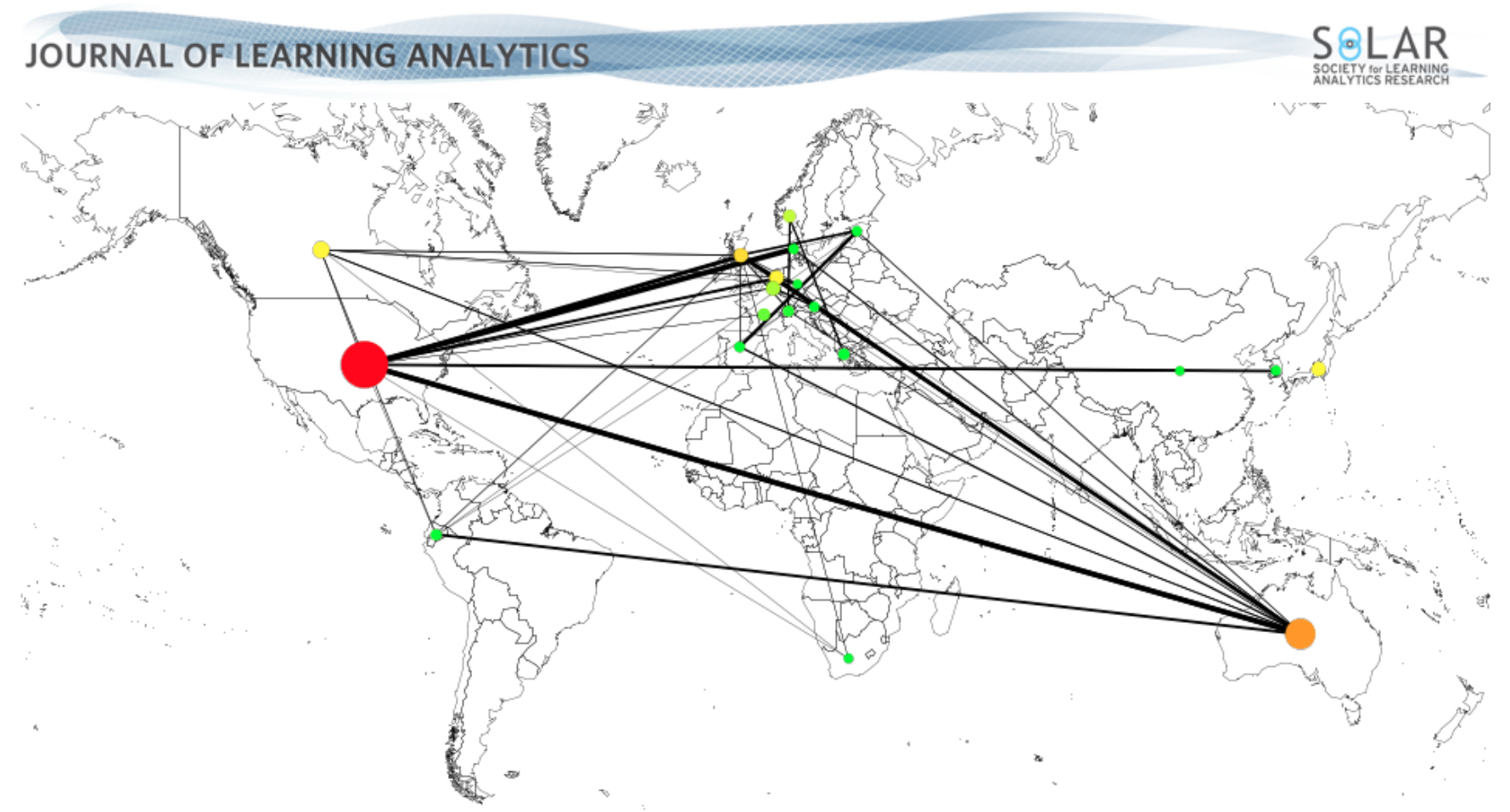

Figure 11. Accepted papers per country. Node's size and color represent the contribution of accepted papers for a given country. The edges represent papers co-authored by authors in two different countries. The width of the edges represents the number of international collaborative papers.

a node represents the "age" of the author in LAK. Green nodes are new authors in LAK, while orange and red nodes are LAK veterans. As it can be seen in the figure, there is a giant component of interconnected authors. Instead of several groups competing with each other, there is a healthy dose of cross-pollination between groups (at least one author is shared among groups). It is also important to note that while there is interconnection between red, orange and yellow nodes, there is also a connections between green and all the other colors. This reflect the openness of LAK contributors to new researchers. Instead of isolated "old" cliques, these older authors are the backbone of an age-independent collaboration networks. The numbers of links between nodes is also a testament of the tendency at LAK of papers produced by bigger groups, reflecting the interdisciplinarity of the field and the need of a large set of expertise in order to tackle Learning Analytics problems.

\section{References for LAK-18}

Analyzing the citations found in the accepted papers, the main references for LAK can be detected. As expected, the main targets of references for accepted LAK-18 papers are the previous LAK conferences. Due to its temathic proximity, the Educational Data Mining (EDM) and Learning at Scale (L@S) conferences are also cited in LAK-18 papers. The Journal of Learning Analytics (JLA), although it is a relatively new and less massive venue, is one of the main references for LAK among journals. Apart from JLA, journals that are more general for the area of Education and Education Technologies are also high in the list, especially if they had a special issue on Learning Technologies. Example of these journals are the Journal of Educational Psychology, Transactions on Learning Technologies and Computers and Education.

\section{Qualitative Analysis of LAK-18}

To complement the quantitative analysis, a more subjective but valuable perspective is presented. This analysis is the result of the work of the Program Committee in the review, meta-review and selection of the program of LAK-18. The most important aspect of this analysis is the identification of trends in the field represented by papers in the conferences and the present special section of JLA.

\section{Trends in LAK-18}

LAK' 18 shows a variety of trends in the accepted papers as illustrated by the session names of the conference. These trends are presented in the following and the relation with topics introduced earlier is discussed at the end of this section. They follow closely, but not completely, the sessions of the conference. The reason for the difference is that sessions of the conference have to follow a timely format to present papers in exactly 90 minutes. For example, four full papers with the same theme cannot belong to the same session; another session has to be found for the fourth paper. Therefore, some sessions like Dashboards, 


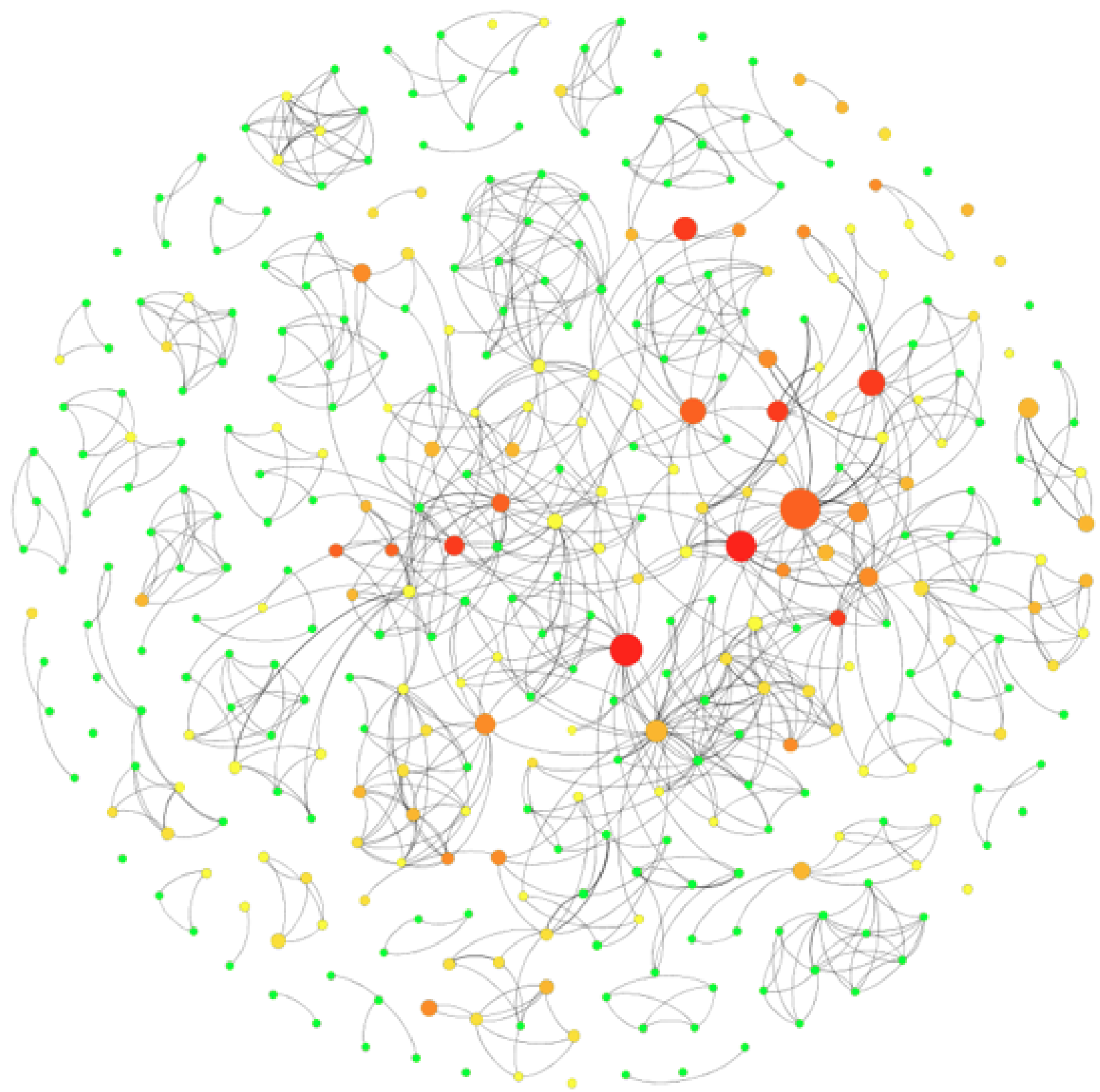

Figure 12. Co-authorship graph. Node's size represents the number of papers submitted to LAK conferences. Node's color represent 'age' in LAK, with green being new authors to LAK and red authors with more than have been accepted to more than 5 conferences. The link represent that both authors collaborated in a LAK-18 paper. 


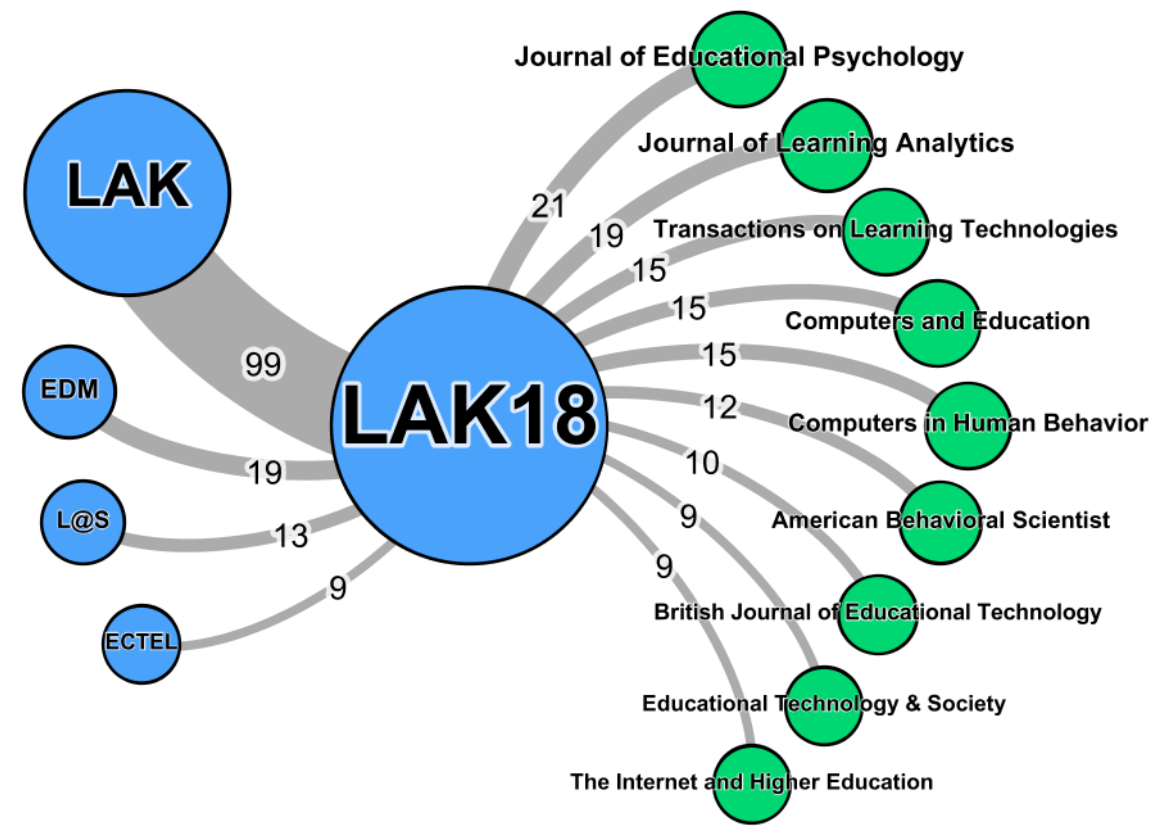

Figure 13. References of accepted LAK-18 papers. Node's size represent the number of citations from accepted LAK-18 papers.

learning design video, group papers with different trends and this session is divided into three trends in the following. As another example, nine full papers with the same theme like Performance Prediction Retention would mean three sessions with the same name. In the conference, this trend was divided into one session called Performance Prediction and two sessions called Retention I and Retention II. Note that some papers on MOOCs attend equally two trends and are counted in the two of them: a paper dealing with retention in MOOCs for example is counted in the trend MOOCs as well as in the trend Performance Prediction Retention.

The first trend which continues to grow strong from the last conferences is Discourse Analysis which this year was declined into three session names: Discourse general, four papers, Discourse Analysis in MOOCs, two papers, and Discourse Analysis in Writing, two papers. Following are two trends both covered by seven papers. One trend is MOOCs, with two papers in a session called Discourse Analysis in MOOCs, and two papers in the session Evaluation Feedback and Retention respectively. The other trend is Performance Prediction Retention, a trend present form the beginning in the LAK conferences. The following trend by the number of papers is User-Centered Design, which matches exactly the theme of LAK- 18.

The next trends follow closely again when counting the number of papers addressing them: five or four papers. They are listed here in alphabetical order: Academic Analytics, Dashboards, Evaluation Feedback, Infrastructure, rather classical trends in the LAK conferences and Multimodal Analytics which continues growing. Finally, the following trends were covered by one or two papers in the LAK-18 conference: Adaptive Learning, Institutional Adoption Policies, Learning Design, Self-regulation, Student Behavior, Theory (three papers) and Videos.

One notices that trends, as presented in this section, and topics, as presented in a previous section, match only partly. An example for matching is the trend infrastructure and the topic Learning Analytics infrastructure in the sense that all accepted papers in the trend infrastructure have also the topic Learning Analytics infrastructure selected by their authors. Another example is the trend Institutional Adoption Policy that merges the two topics Adoption Strategies and Learning Analytics Policies. The trend Performance Prediction Retention encompasses the topic Retention Students at Risk. Other trends are not found as such in the topics. Topics have been defined a few years ago to reflect trends in the conference at that time. New themes like MOOCs or Multimodal Analytics are not included in the topics yet. Some topics like Use and Evaluation of Learning Analytics Tools appear now too general. For example, all accepted papers in the dashboard trend have Use and Evaluation of Learning Analytics Tools as a selected topic, and two have feedback systems, but otherwise no topic in common. The topic Understanding Discourse, associated with Natural Language Processing, is more narrow that the identified trend Discourse Analysis. It might be worthwhile considering to realign trends, as found in the recent conferences, and topics as defined in Easychair. 
Selected Papers

This special issue contains eight papers. From these eight papers, three were originally submitted as short papers to the conference and the other as full papers. The top-five contributors of this special issue are: USA (7 authors), the UK (5), the Netherlands (5), Australia (4) and Norway (3), which reflects to some extend the main trends in the submissions. Interestingly, five of these papers have authors coming from at least two countries, reflecting here again the internationality of LAK. Four of these papers had Use and Evaluation of Learning Analytics Tool and also four had Learning / Instructional Design as selected topics when submitted to LAK, these two most often selected topics.

The papers of this issue cover seven trends from the 15 listed in the preceding section. Following the order given above, the first trend to be covered is MOOCs with the paper:

- Scaling Effective Learning Strategies: Retrieval Practice and Long-Term Knowledge Retention in MOOCs by Dan Davis, René Kizilcec, Claudia Hauff, Geert-Jan Houben. This paper reports about deploying an Adaptive Retrieval Practice System in a MOOC. Contrarily to their expectations, the authors observed no effect on the learning outcomes and discuss this issue.

Two papers address the trend Academic Analytics:

- Taken Together: Conceptualizing students' concurrent course enrollment across the post-secondary curriculum using temporal analytics by Michael Brown, R. Matthew DeMonbrun, Stephanie Teasley. This paper analyze the impact of co-enrollment in the academic risk of students and their chances to get out-of-risk.

- Learn From Your (Markov) Neighbor: Coenrollment, Assortativity, and Grade Prediction in Undergraduate Courses by Joshua Patrick Gardner, Christopher Brooks, Warren Li. This paper also uses the the co-enrollment information to improve the predition of student performance.

The next trend Dashboard is covered by the paper:

- Exploratory versus Explanatory Visual Learning Analytics: Driving Teachers' Attention through Educational Data Storytelling by Vanessa Echeverria, Roberto Martinez-Maldonado, Simon Buckingham Shum, Katherine Chiluiza, Roger Granda, Cristina Conati. This paper proposes to use "Educational Data Storytelling" to enrich dashboards with explanations in line with the intended learning design.

Evaluation Feedback is attended by two papers; the first one is mentioned above as it also addresses the trend MOOCs:

- Scaling Effective Learning Strategies: Retrieval Practice and Long-Term Knowledge Retention in MOOCs by Dan Davis, René Kizilcec, Claudia Hauff, Geert-Jan Houben.

- Topic Dependency Models: Graph-Based Visual Analytics for Communicating Assessment Data by Hassan Khosravi, Kendra Cooper. This paper proposes a collection of Topic Dependency Models as a graphical visualisation and explains their definition, calculation and potential for instructors in particular to visualize assessment data in courses.

The trend Multimodal Analytics is covered by the following paper:

- Gaze-Driven Design Insights to Amplify Debugging Skills: A Learner-Centered Analysis Approach by Katerina Mangaroska, Kshitij Sharma, Michail Giannakos, Hallvard Trætteberg, Pierre Dillenbourg. The authors of this paper analyse data coming from two heterogeneous sources: gaze data and interactions data in a mirroring tool integrated in the eclipse programming environment. The analysis show that different patterns distinguish experts and novice students.

The trend Institutional Adoption Policies is covered by one paper:

- The SHEILA framework: informing institutional strategies and policy processes of learning analytics by Yi-Shan Tsai, Pedro Manuel Moreno-Marcos, Ioana Jivet, Maren Scheffel, Kairit Tammets, Kaire Kollom, Dragan Gašević. This paper presents a complete framework to develop Learning Analytics policies in Higher Education Institutions.

Finally, Learning Design is covered by:

- Using temporal analytics to detect inconsistencies between learning design and students' behaviours by Quan Nguyen, Michal Huptych, Bart Rienties. This study investigates to what extent students' timing of engagement aligned with instructor learning design, and how engagement varied across different levels of performance. 


\section{Conclusions}

The Learning Analytics and Knowledge conference has become the main venue to observe and present the main advances in the field of Learning Analytics. As such, it is the ideal point to measure the pulse of the field and its research community. From the quantitative analysis performed with the conference data, it is clear that LAK is maturing and growing at a sustainable pace. The review process of the conference, while difficult due to its interdisciplinary nature, is possible due to a large set of reviewers and senior reviewers. The inclusion of the meta-review phase as well as the double-blind review process this year has helped the conference selection process to be fairer than previous editions. The learning analytics community is growing rapidly due to its open and collaborative and international nature. Finally, citation analysis proves that LAK is creating its own body of knowledge that is references in the new papers each year. LAK is also one of the most cited venues in the Educational Technology field. The qualitative analysis performed by the chairs of the conference to assemble the program identified several trends, represented by exemplary articles in this special section. From Discourse to Multimodal Learning Analytics, the field is expanding and specializing.

We wish you a pleasant and enriching read!

\section{References}

Suthers, D., \& Verbert, K. (2013). Learning analytics as a "middle space". In Proceedings of the third international conference on learning analytics and knowledge (pp. 1-4). New York, NY, USA: ACM. https://dx.doi.org/10.1145/2460296.2460298 